\title{
Measurement of Frequency Dependent Radiation Patterns of Ultrasonic (Wedge) Transducers
}

\author{
$\underline{P . P l O B}^{1}$, S. J. Rupitsch ${ }^{1}$, R. Lerch $^{1}$ \\ ${ }^{1}$ Chair of Sensor Technology, University of Erlangen-Nuremberg, \\ Paul-Gordan-Str. 3/5, 91052 Erlangen, Germany \\ peter.ploss@fau.de
}

\begin{abstract}
It is well known from theory that a transducer's radiation pattern depends on the aperture and driving frequency. Furthermore, it is widely assumed that the main lobe's orientation does not change with frequency. This work presents a novel measurement method for quickly obtaining the frequency resolved radiation pattern of ultrasonic (wedge) transducers (UT). The setup comprises a water basin, where an UT is mounted on an angle controlled tilting unit. A pulsed excitation is applied to the UT and the emitted acoustic wave is received by the same UT after being reflected at a fixed reflector plate. The received pulse is then analyzed by means of Fourier decomposition. Repetition for different angles yields a two-dimensional map angle vs. frequency. It is shown that piston-type transducer's behavior matches theoretical models very well. Wedge transducers though exhibit a frequency dependent change of the dominant radiation angle, which is not described in literature. Similar results are obtained by attaching a small plate to the wedge transducer.
\end{abstract}

Key words: ultrasound, transducer, frequency dependent directivity pattern, model

\section{Introduction}

Determination of the characteristics of ultrasonic (wedge) transducers (UT) has been subject to many theoretical and practical studies in the last decades [1, 2, 3]. Precise knowledge of an UT's behavior in a given operating condition for both spatial and time domain is often crucial. Wrong assumptions lead to measurement deviations, as is for example demonstrated in the context of industrial Clamp-on flow measurements [4]. Thus, the need for theoretical modeling and practical validation is obvious.

This work first describes the fundamental theory, needed for modeling ultrasonic (wedge) transducers regarding their radiation behavior. Afterwards, the measurement setup for determination of UT's frequency dependent radiation patterns is presented. The analysis of measurement data, based on the Fourier Transform, is discussed in the subsequent section. Lastly, the change of radiation behavior when a thin metal plate is mounted on a wedge transducer is reviewed.

\section{Theory}

Typical figures of merit of an UT are center frequency, bandwidth, aperture (size of the active element) and angle of divergence. It is important to note that the first two parameters are associated with the time domain and the remaining two with the spatial domain.

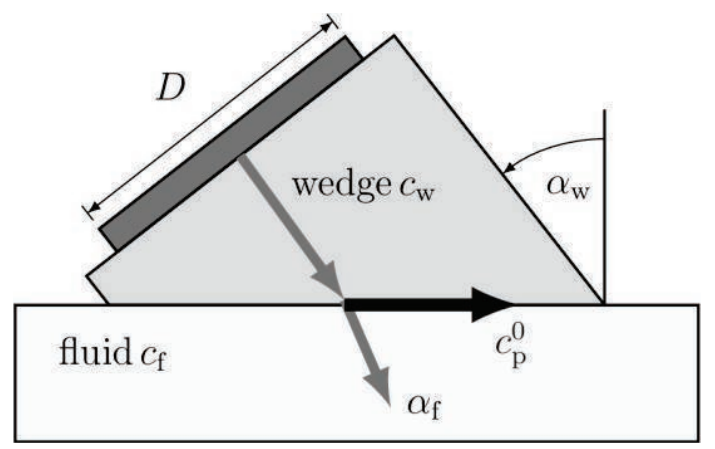

Fig. 1. Sketch of ultrasonic wedge transducer with angle of incidence $a_{\mathrm{w}}$ and speed of sound in wedge $c_{w}$. A piezoceramic disc with diameter $D$ is the active element and it is glued onto the wedge. The transducer is slightly immersed in a fluid with speed of sound $c_{f}$.

The nominal phase velocity $c_{\mathrm{p}}^{0}$ on the interface of a wedge transducer (Fig. 1) is derived from the wedge sound velocity $c_{\mathrm{w}}$ and wedge angle $\alpha_{\mathrm{w}}$ by $c_{\mathrm{p}}^{0}=c_{\mathrm{w}} / \sin \left(\alpha_{\mathrm{w}}\right)$.

The theoretical angle of refraction in the adjacent fluid $\alpha_{\mathrm{f}}$ is calculated using Snell's law

$\alpha_{\mathrm{f}}=\sin ^{-1}\left(\frac{c_{\mathrm{f}}}{c_{\mathrm{p}}^{0}}\right)$. 


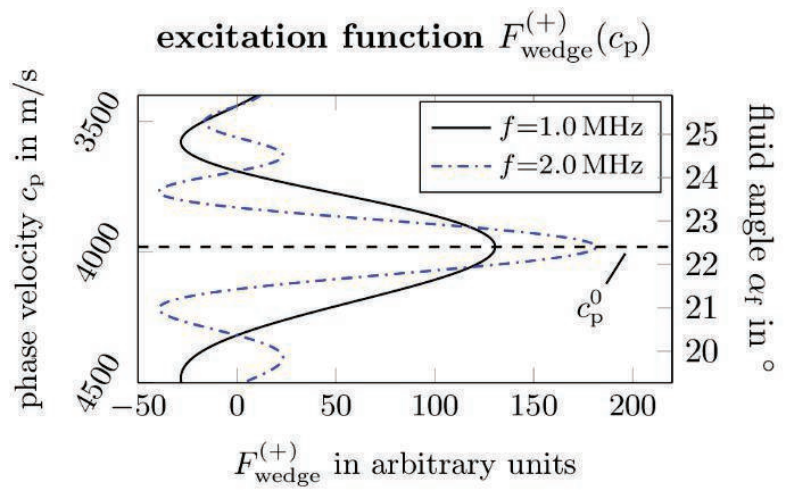

(a)

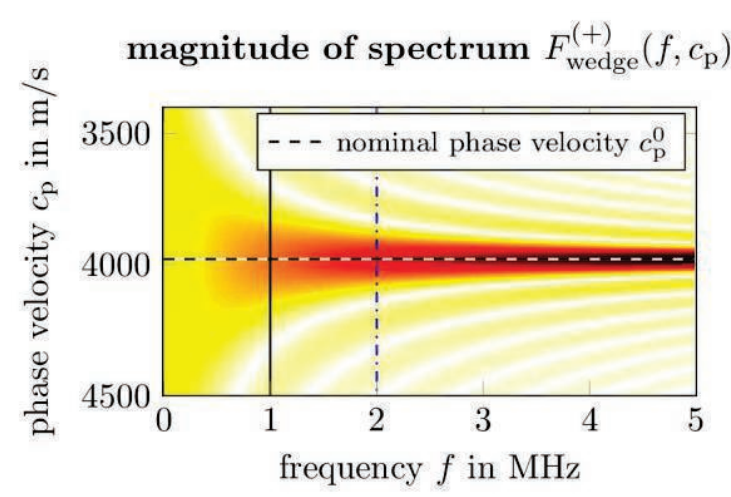

(b)

Fig. 2. Theoretical excitation function of wedge transducer for two distinct frequencies $f(a)$. Resulting excitation magnitude spectrum (b) for frequency range (arbitrary units, normalized to unit power per frequency).

It coincides with the expected dominant propagation direction of the sound in the fluid $\alpha_{\mathrm{f}, \mathrm{dom}}$.

Due to the finite size of the sound source, it is impossible to generate plane waves with a single wave vector $\vec{k}$, meaning that sound radiation always takes place for a range of directions. This is commonly referred to as the directivity pattern.

A compact formulation for calculation of the radiation pattern of ultrasonic wedge transducers of finite size is detailed in [5, Eq. (14.30)]

$F_{\text {wedge }}^{(+)}(k)=\frac{D}{\cos \alpha_{\mathrm{w}}} \operatorname{sinc}\left(\frac{\left(k-k_{\mathrm{w}} \sin \alpha_{\mathrm{w}}\right) D}{2 \cos \alpha_{\mathrm{w}}}\right)$.

This excitation function and takes the diameter of the piezoceramic disc $D$, the shoe angle $\alpha_{\mathrm{i}}$ (angle of incidence), wave number in the wedge $k_{\mathrm{w}}=\omega / c_{\mathrm{w}}=2 \pi f / c_{\mathrm{w}}$ and the variable wave number $k=\omega / c_{\mathrm{p}}$ as parameters. It is derived by the assumption of a uniform pressure distribution on the surface of the piezoceramic disc.

Figure $2 \mathrm{a}$ shows the theoretical excitation function of a wedge transducer for two distinct frequencies. For both frequencies, the strongest excitation is achieved at the nominal phase velocity $c_{\mathrm{p}}^{0}$, which is mapped to the dominant fluid angle $\alpha_{\mathrm{f}, \mathrm{dom}}$ by Eq. 2. Contrary to a piston transducer, the locations of the side lobes are not symmetric with respect to that dominant angle. Computation of the excitation function for a variety of frequencies leads to the magnitude spectrum, depicted in Fig. $2 \mathrm{~b}$. It is normalized to contain unit power per frequency $f$. Obviously for higher frequencies, directivity increases. For low frequencies, radiation is almost uniform across a wide range of angles. As can be seen from Eq. 3, directivity can also be influenced by both the shoe angle $\alpha_{\mathrm{w}}$ and the diameter of the piezoceramic disc $D$.

\section{Measurements}

In order to verify calculated radiation patterns, a measurement setup consisting of a water basin with a computer controlled tilt stage is utilized (Fig. 3). Both piston type and wedge transducers of different center frequencies and aperture can be examined by mounting them on a transducer fixture. The inclination of the transducer is altered by an electronically adjustable goniometer. The actual inclination $\alpha_{\mathrm{i}, \text { actual }}$ is controlled by means of a high-precision absolute angle inclination sensor with an accuracy better $0.05^{\circ}$.

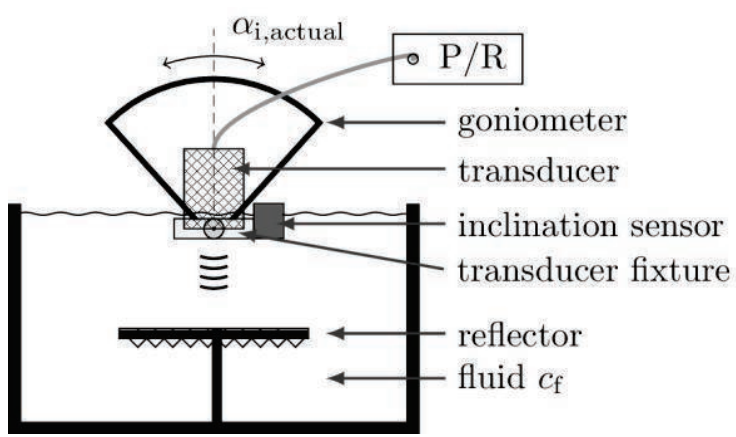

Fig. 3. Sketch of measurement setup with tiltable ultrasonic (wedge) transducer.

For a measurement series, typically an angular range of several degrees and an angular resolution of $0.05^{\circ}$ is chosen. This results in an overall measurement time of about twenty minutes.

The transducer is electrically connected to a pulser/receiver 5800PR from Olympus. For a given inclination, the transducer is excited with a broadband pulse. The wave packet is received and digitized by an oscilloscope after being reflected at the fixed reflector. The effective resolution and signal-to-noise ratio can be easily increased by averaging several data acquisitions. 


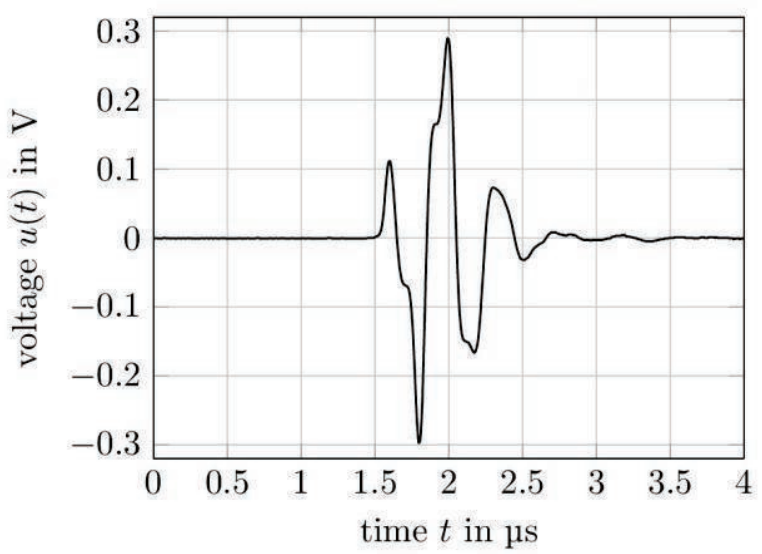

(a) time domain

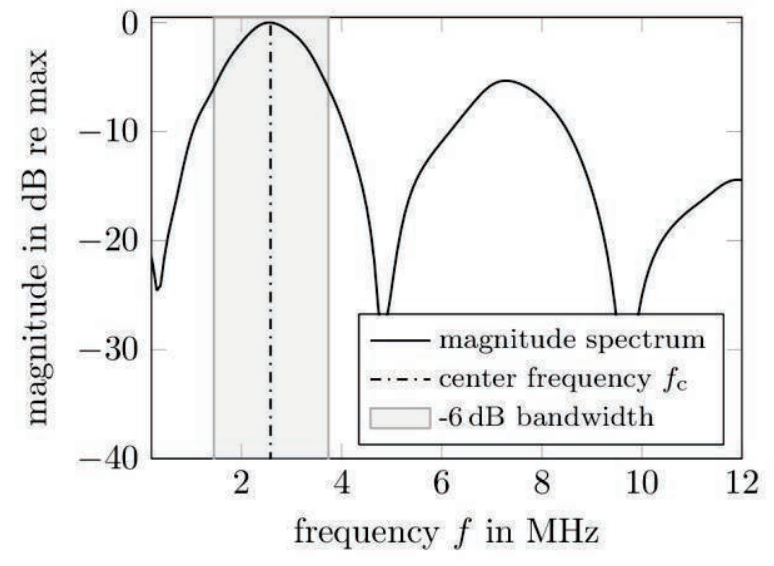

(b) frequency domain

Fig. 4. Acquired voltage signal (a) from a pulse-echo measurement of a NDT transducer H2K with center frequency $f_{c}=2.25 \mathrm{MHz}$ at inclination $\alpha_{i, \text { actual }}=0^{\circ}$. Thereof calculated single-sided magnitude spectrum $|\underline{U}(f)|$ (b).

\section{Analysis of Measurement Data}

Figure 4a shows the received pulse-echo signal $u(t)$ of a piston type transducer $H 2 K$ from Krautkrämer (today GE Sensing \& Inspection Technologies) with nominal center frequency $f_{\mathrm{c}}=2.25 \mathrm{MHz}$. The voltage signal $u(t)$ is proportional to sound pressure in the fluid. The magnitude spectrum $|\underline{U}(f)|$ (Fig $4 b)$ reveals the transducer's center frequency and $-6 \mathrm{~dB}$ bandwidth. Analysis of the peak-to-peak voltage $u_{\mathrm{pk} 2 \mathrm{pk}}\left(\alpha_{\mathrm{i}, \text { actual }}\right)$ for a range of inclination angles $\alpha_{i, a c t u a l}$ gives an idea of the transducer's radiation pattern. Regression analysis with a parabola as target function yields the direction of strongest radiation $\alpha_{\mathrm{i}, \max }=0.09^{\circ}$ (Fig. 5). Since the mechanical construction of the measurement setup does not permit perfect levelling of the fixture with respect to the reflector, this angle serves as the reference for further measurements for all investigated transducers.

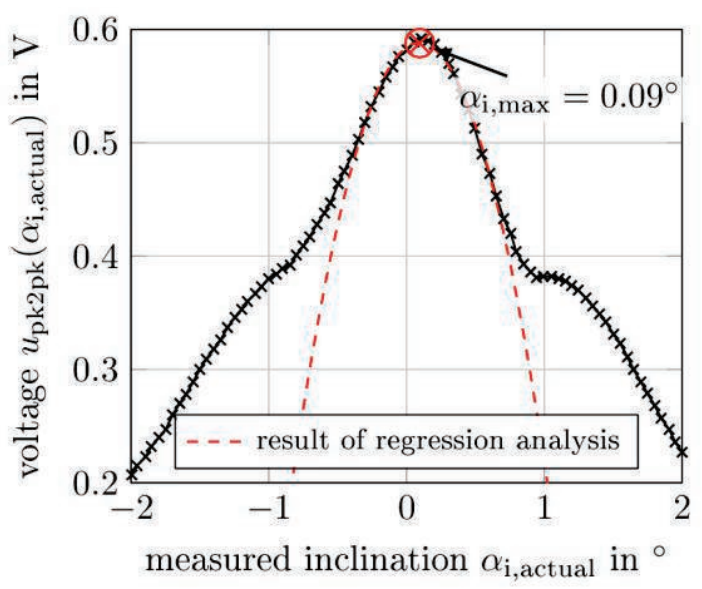

Fig. 5. Determination of the angle with strongest sound radiation of a $\mathrm{H} 2 \mathrm{~K}$ transducer. A regression analysis is applied to the peak-to-peak voltages, obtained for different inclination angles.
In order to metrologically obtain the frequency resolved radiation pattern of a transducer, the signal $u(t)$ is acquired for a range of inclination angles $\alpha_{i, \text { actual }}$. By applying the Fourier transform to each of those waveforms and squaring the result, an angle dependent power spectral density $S_{\mathrm{xx}}(f)=|\underline{U}(f)|^{2}$ is calculated (Fig. 7a). As expected, most energy is present in the vicinity of the center frequency $f_{\mathrm{c}}$ and strongest radiation direction $\alpha_{\mathrm{i}, \max }$. The dashed line coincides with the magnitude spectrum of Fig. 4b. Normalizing the data to maximum power for each frequency reveals the frequency dependent radiation pattern (Fig $7 \mathrm{~b}$ ). Normalizing to maximum instead of overall power per frequency (Fig. 2b) is done for increased visual contrast. Due to the notches in the magnitude spectrum at around $4.9 \mathrm{MHz}$ and 9.8 $\mathrm{MHz}$ (Fig. 4b), the data appears noisy at those frequencies.

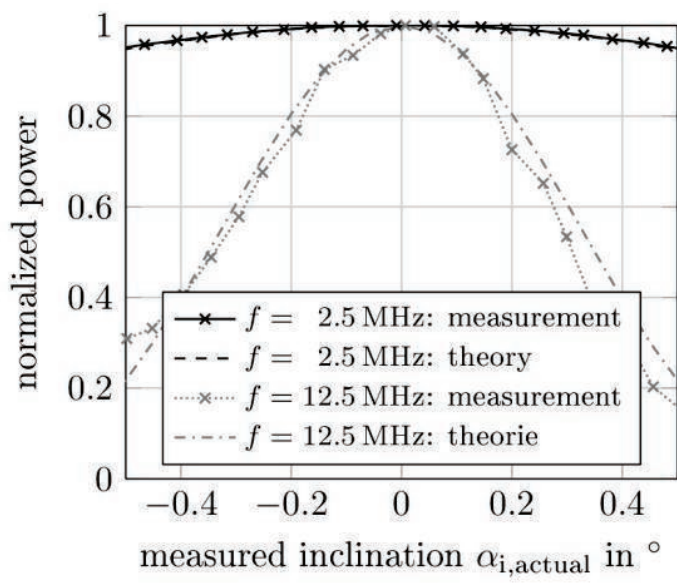

Fig. 6. Radiation pattern of a H2K transducer with nominal center frequency $f_{c}=2.25 \mathrm{MHz}$ for two different frequencies. Comparison between theory (Eq. 4) and measurement (Fig. 7b). 


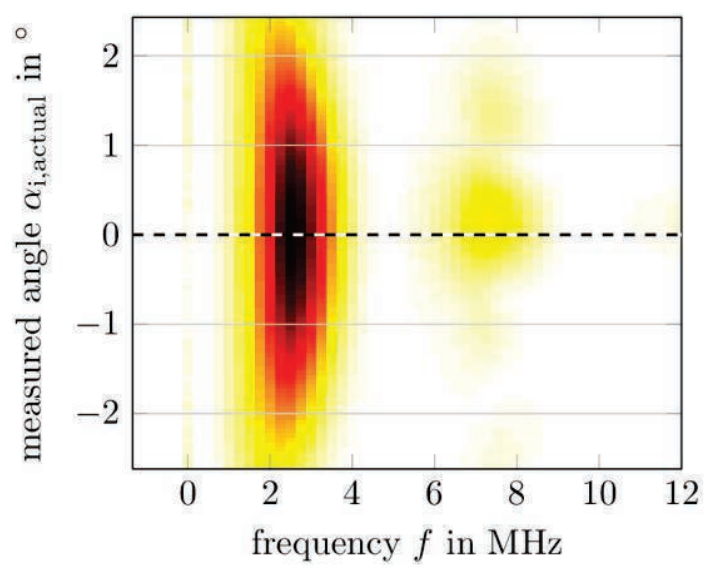

(a) normalized to global maximum

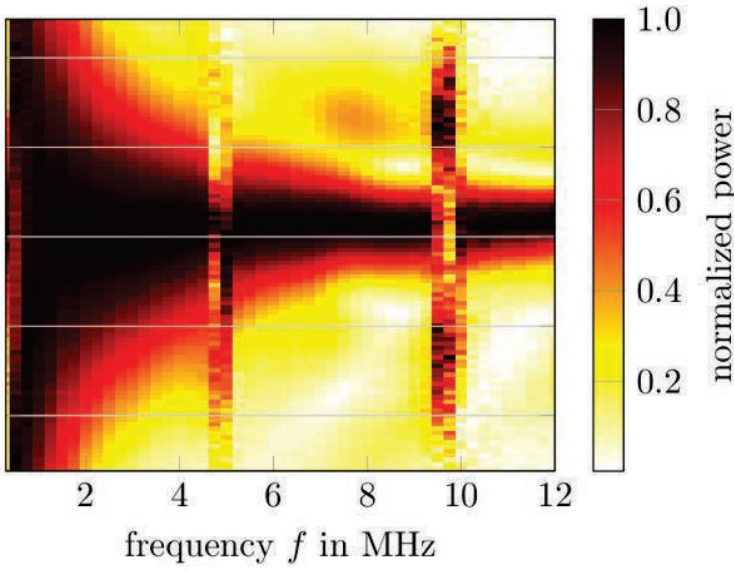

(b) normalized to maximum per frequency

Fig. 7. Power spectral density $S_{x x}(f)$ calculated from many pulse echo measurements at different inclination angles (a). Scaling to the maximum per frequency reveals the frequency dependent radiation pattern (b).

The frequency dependent farfield radiation pattern of the piston source $\mathrm{H} 2 \mathrm{~K}$ with aperture radius $r=5 \mathrm{~mm}$, polar angle $\Theta$ and Bessel function $J_{1}$ is calculated by [6, Eq. 2.106]

$R(f, \Theta) \propto \frac{J_{1}(k r \sin (\Theta))}{k r \sin (\Theta)}$.

As shown in Fig. 6, theory matches the measured radiation pattern very well, both near the center frequency ( $f=2.5 \mathrm{MHz})$ and even far away $(f=12.5 \mathrm{MHz})$, where the measurement signals $u(t)$ carries little power, due to the limited transducer bandwidth. Measurement data is shifted by the frequency dependent direction of strongest radiation $\alpha_{\mathrm{i} \text { max }}(f)$, so that the maxima coincide with the calculated curves. Those angles $\alpha_{\mathrm{i}, \max }(f)$ are determined by means of a regression analysis, like above.

This result confirms that the proposed measurement setup allows precise determination of the frequency dependent radiation pattern.

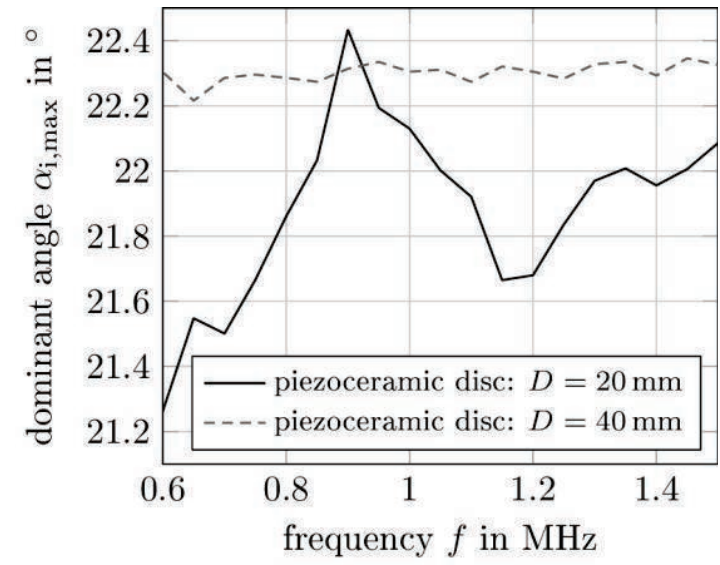

Fig. 8. Dominant radiation angle $\alpha_{i, \max }(f)$ of two wedge transducers with different aperture size.

\section{Results}

Figure 8 presents the frequency dependent dominant radiation angle $\alpha_{\mathrm{i} \text { max }}(f)$ of two different wedge transducers with center frequency $f_{\mathrm{c}}=1 \mathrm{MHz}$. They are constructed with the same shoe angle $\alpha_{\mathrm{i}}$ but their aperture size is different. As expected, the radiation angle does hardly change over frequency in case of the transducer with the large piezoceramic disc. Surprisingly, this is not true for the transducer with the smaller aperture. The angle of the dominant radiation direction changes about $1^{\circ}$ in vicinity of the center frequency. This undesired effect is responsible for measurement deviations, in case of clamp-on flow measurements [4]. The effect is mitigated by either enlarging the active element or increasing the center frequency (by reducing the discs thickness). Principally, the observed phenomenon is caused by geometric dispersion in the wedge [3]. Phase velocity $c_{\mathrm{w}}$ is dispersive, since the wavelength is in the same magnitude of the geometric dimensions [7, p. 116ff. and 470ff.].

Now, a plate with thickness $d_{\mathrm{w}}=5.5 \mathrm{~mm}$ is attached to the small transducer by a thin layer of couple gel. This setup yields a very strong dependence of the dominant radiation angle of almost $4^{\circ}$ (Fig. 9). Consequently, in context of ultrasound based flow-measurement, large measurement deviations will arise, when the system is operated with narrowband excitation signals, like windowed sine bursts at some frequency. This behavior is caused by the generation of different Lamb wave modes that involve different phase velocities. A change of phase velocity $c_{\mathrm{p}}$ from the nominal phase velocity $c_{\mathrm{p}}^{0}$ directly translates into a change of the fluid angle $\alpha_{\mathrm{f}}$, as is described by Eq. 2 . 


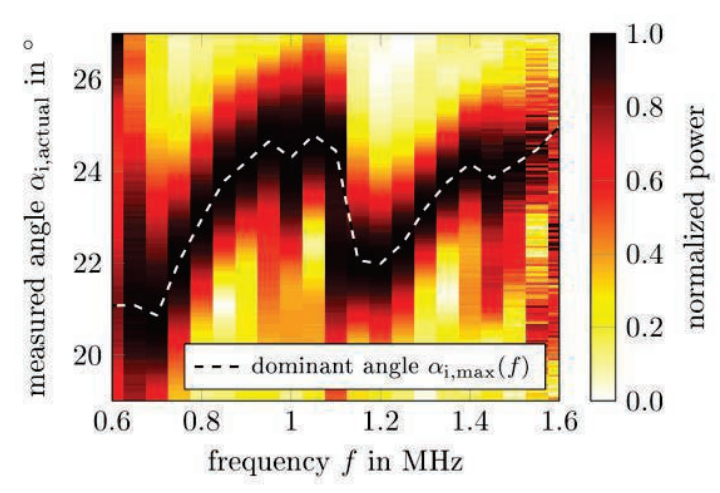

Fig. 9. Strong frequency dependence of the dominant radiation angle $\alpha_{i, \max }(f)$, when a wedge transducer $(D=20 \mathrm{~mm})$ is mounted on a $5.5 \mathrm{~mm}$ thick plate.

\section{Conclusion}

This work describes a novel measurement setup for the fast determination of transducers' frequency dependent radiation pattern. It is based on the spectral analysis of pulse-echo waveforms that are acquired at different tilt angles of the transducer under test.

By comparing the theoretical farfield radiation pattern of a piston type transducer with data obtained from measurements, the suitability of the approach is proven.

Analysis of a wedge transducer with large aperture confirms the theoretical prediction of constant dominant radiation angle over frequency (Eq. 3, Fig 2a). A scaled version of that transducer with smaller piezoceramic disc and wedge though exhibits a frequency dependence of that angle, which is attributed to geometric dispersion.

Attachment of a plate to that wedge transducer leads to an even stronger change of the prevalent radiation angle. This phenomenon is caused by generation of lamb waves in the plate and the accompanying change of phase velocity. Future work will concentrate on a model for predicting the described frequency dependence of the fluid angle.

\section{References}

[1] J. Krautkrämer, H. Krautkrämer, Springer, Werkstoffprüfung mit Ultraschall (1986)

[2] E. Williams, Academic Press, Fourier Acoustics (1999)

[3] P. Ploß, S. J. Rupitsch, P. Kroh, R. Lerch, Sensors and Measuring Systems 2014; 17. ITG/GMA Symposium; Proceedings of, Frequency Dependent Sound Radiation of Ultrasonic Wedge Transducers, 2014

[4] P. Ploß, S. J. Rupitsch, R. Lerch, IEEE Sensors Journal 14(7), Extraction of Spatial Ultrasonic Wave Packet Features by Exploiting a Modified Hough Transform, 2389-2395 (2014); doi: 10.1109/JSEN.2014.2311160
[5] J. L. Rose, Cambridge University Press, Ultrasonic Waves in Solid Media (1999)

[6] E. G. Williams, Academic Press, Fourier Acoustics: Sound Radiation and Nearfield Acoustical Holography (1999)

[7] K. F. Graff, Dover Publications, Wave Motion in Elastic Solids, ser. Dover Books on Engineering Series (1975) 